\title{
High-Pressure Effect on Structural and Magnetic Properties of $\mathrm{MnS}$
}

\author{
A. $\operatorname{Khaldi}^{a, b, *}$, A. Gueddim ${ }^{c}$, N. Bouarissa ${ }^{d}$ And L. TABourot ${ }^{e}$ \\ ${ }^{a}$ Laboratory of Materials and Electronic Systems (LMSE), University of Mohamed El Bachir El Ibrahimi, 34000, \\ Bordj Bou Arreridj, Algeria \\ ${ }^{b}$ Department of Civil Engineering, Faculty of Science and Technology, University of Bordj Bou Arreridj, \\ 34000, Algeria \\ ${ }^{c}$ Materials Science and Informatics Laboratory, Faculty of Science, University of Djelfa, \\ 17000, Djelfa, Algeria \\ ${ }^{d}$ Laboratory of Materials Physics and Its Applications, University of M'sila, 28000 M'sila, Algeria \\ ${ }^{e}$ Université Savoie Mont Blanc, SYMME, F-74000 Annecy, France

\begin{abstract}
The present contribution deals with the structural and magnetic properties of MnS at normal and highpressure. The calculations are performed using the pseudopotential plane-wave method within the generalized gradient approximation. The non-magnetic and antiferromagnetic spin arrangements are examined. The effect of pressure on the lattice parameter and magnetic moment is analyzed and discussed. At zero pressure, our findings are found to be in reasonably good accord with experiment. All features of interest show a monotonic behavior
\end{abstract} \\ under applied pressure.
}

DOI: $10.12693 /$ APhysPolA.137.483

PACS/topics: structural properties, magnetic properties, MnS

\section{Introduction}

Due to their interesting fundamental properties which include optical, transport, and magnetic properties, bulk chalcogenides materials have attracted great interest [1-9]. MnS is a semiconductor which has technological applications in solar cell coatings and in several other optoelectronics [2]. The material of interest was grown in the cubic zinc-blende structure $(\beta-\mathrm{MnS})$ in bulk [1].

The knowledge of the structural and magnetic properties of semiconductors is important for device design and fabrication. The investigation of the behavior of these properties under pressure has become an important subject both theoretically and experimentally [10-19]. This is essentially due to the experimental advances and reliable computational methods for band structure calculations.

In spite of the importance of the fundamental properties of MnS, only about them limited data are published, to the best of our knowledge [2,4]. In this regard, the present contribution deals with the structural and magnetic properties of $\mathrm{MnS}$ semiconductor material in the zinc-blende structure at zero and under applied pressure. The aim of this work is to see how the properties of interest behave under applied pressure.

*corresponding author; e-mail: a.khaldi@univ-bba.dz

\section{Computational details}

Computations are carried out using the full-potential linearized augmented plane wave (FP-LAPW) method as implemented in the WIEN2K code [20], based on the density functional theory (DFT) [21]. The spin polarized calculations are performed with both spin-up and spin down channels. The exchange-correlation potential is described using the generalized gradient approximation (GGA) of $\mathrm{Wu}$ and Cohen (WC-GGA) [22]. In the FP-LAPW method, each unit cell is divided into nonoverlapping muffin-tin (MT) spheres of radii $R_{\mathrm{MT}}$ and an interstitial region.

A plane wave cutoff of $R_{\mathrm{MT}} \mathrm{K}_{\max }=8$ (where $K_{\max }$ is the cut-off of the plane waves) is used in the present calculation. The $R_{\mathrm{MT}}$ values are chosen to be 2.1 a.u. for $\mathrm{Mn}$ and 1.8 a.u. for S. The $k$ integration over the Brillouin zone is performed using the Monkhorst and Pack scheme [23], where a grid of $10 \times 10 \times 10$ is considered.

\section{Results and discussion}

Figure 1 shows the variation in the total energy versus the volume for $\mathrm{MnS}$ in the zinc-blende structure in the antiferromagnetic (AFM) and non-magnetic (NM) spin arrangements. By observing Fig. 1, it is obvious that the AFM zinc-blende $\mathrm{MnS}$ is more stable than NM zincblende $\mathrm{MnS}$.

The variation in the total energy as a function of the volume for $\mathrm{MnS}$ has been computed. The obtained curves are fit to the Murnaghan equation of state. This has allowed the determination of the equilibrium structural 


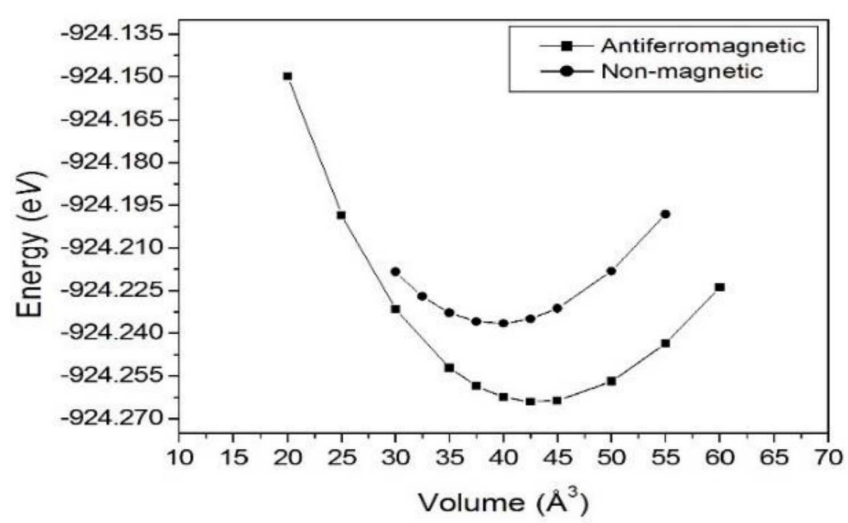

Fig. 1. Total energy as a function of volume for zincblende $\mathrm{MnS}$ in the AFM and NM spin arrangements.

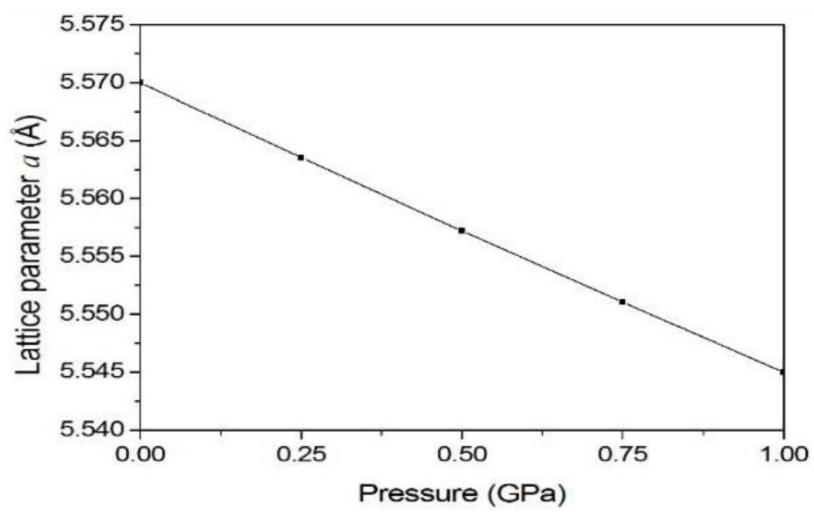

Fig. 2. Lattice parameter as a function of pressure in zinc-blende $\mathrm{MnS}$.

parameters such as the lattice parameter $a_{0}$, the bulk modulus $B$ and its first-pressure derivative $B^{\prime}$ similarly to the works reported in Refs. $[24,25]$. Our findings yielded values of $a_{0}=5.57 \AA, B=71.2 \mathrm{GPa}$, and $B^{\prime}=5.8$ for zinc-blende $\mathrm{MnS}$. The experimental value of $a_{0}=5.60 \AA$ quoted in Ref. [26] seems to deviate from our obtained value by less than $1 \%$. On the other hand, recently Kavci and Cabuk [2] have calculated $a_{0}, B$, and $B^{\prime}$ for zinc-blende $\mathrm{MnS}$ using the norm-conserving pseudopotentials method within the local spin density approximation. Their results yielded values of $a_{0}=5.598 \AA$, $B=64.486 \mathrm{GPa}$, and $B^{\prime}=3.372$. Although their value of $a_{0}$ agrees well with our calculated one, our obtained values of $B$ and $B^{\prime}$ appear to be somewhat larger than theirs.

The evolution of $a_{0}$ as a function of pressure for zincblende $\mathrm{MnS}$ is illustrated in Fig. 2. Note that as pressure is increased, the lattice parameter decreases. The behavior is monotonic and almost linear. In fact, the enhancement of pressure leads to the presence of more cloud overlap of electrons which increases the amount of charges migrating from the cation $\mathrm{Mn}$ to the anion $\mathrm{S}$ [27]. This in turn decreases the bond length and hence reduces the lattice parameter.

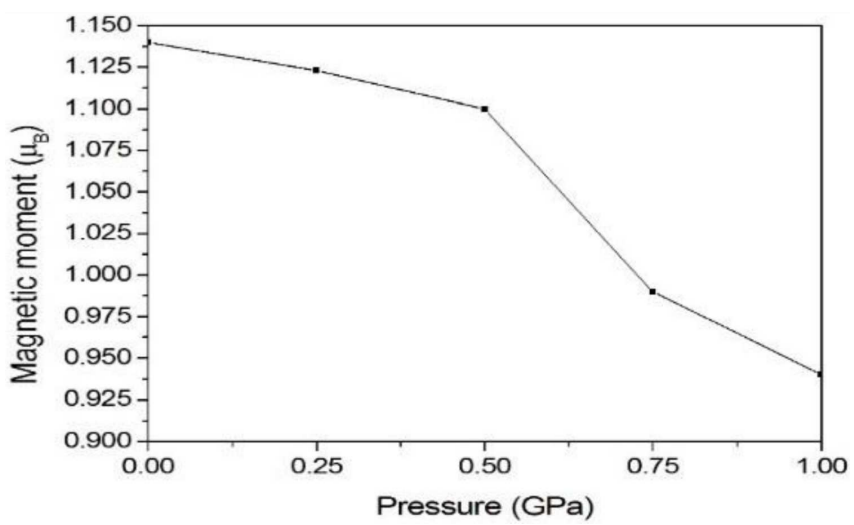

Fig. 3. Total magnetic moment as a function of pressure in zinc-blende MnS.

The total magnetic moment is an important parameter in the study of magnetic properties [28]. This parameter has been computed at various pressures ranging from 0 to $1 \mathrm{GPa}$ (pressures that cover the full stability range of the zinc-blende structure). The pressure dependence of the total magnetic moment for $\mathrm{MnS}$ in the zinc-blende structure is displayed in Fig. 3. We observe that as pressure is enhanced from 0 to $1 \mathrm{GPa}$, the total magnetic moment decreases monotonously and in a non-linear way.

\section{Conclusion}

To summarize, the pressure dependence of the structural and magnetic properties of zinc-blende $\mathrm{MnS}$ was investigated using the FP-LAPW method in the frame work of the DFT in the GGA approach. Our results showed that the AFM zinc-blende MnS is more stable than NM zinc-blende MnS. The behavior of the lattice parameter and total magnetic moment versus pressure was examined and discussed. Our findings showed that the studied features are significantly affected by pressure.

\section{References}

[1] N.A. Spaldin, in: Magnetic Materials: Fundamentals and Applications, 2nd ed., Cambridge University Press, Cambridge 2011 and references therein.

[2] O. Kavci, S. Cabuk, Comput. Mater. Sci. 95, 99 (2014).

[3] W. Zhou, S. Wu, S. Li, J. Magn. Magn. Mater. 395, 166 (2015).

[4] A. Khaldi, N. Bouarissa, L. Tabourot, J. Supercond. Nov. Magn. 31, 1643 (2018).

[5] A. Khaldi, N. Bouarissa, H. Ghodbane, L. Tabourot, Physica B 553, 6 (2019).

[6] R. Basu, S. Bhattacharya, R. Bhatt, A. Singh, D.K. Aswal, S.K. Gupta, Emerg. Mater. Res. 1, 306 (2012).

[7] D.B. Moore, M.J. Stolt, R. Atkins, L. Sitts, Z. Jones, S. Disch, M. Beekman, D.C. Johnson, Emerg. Mater. Res. 1, 292 (2012). 
[8] L. Baruah, S.S. Nath, Emerg. Mater. Res. 2, 186 (2013).

[9] P.R. Kharangarh, G.E. Georgiou, K.K. Chin, Emerg. Mater. Res. 3, 106 (2014).

[10] G.J. Ackland, Rep. Prog. Phys. 64, 483 (2001) and references therein.

[11] S. Saib, N. Bouarissa, P. Rodriguez-Hernández, A. Muñoz, Physica B 403, 4059 (2008).

[12] J.V. Badding, Annu. Rev. Mater. Sci. 28, 631 (1998) and references therein

[13] N. Bouarissa, Phys. Status Solidi B 231, 391 (2002).

[14] A. Mujica, A. Rubio, A. Muñoz, R.J. Needs, Rev. Mod. Phys. 75, 863 (2003).

[15] N. Bouarissa, Mater. Chem. Phys. 73, 51 (2002).

[16] C.S. Mishra, G. Palai, Optik 126, 4685 (2015).

[17] K. Daviau, K.K.M. Lee, Crystals 8, 217 (2018).

[18] S. Daoud, N. Bioud, N. Bouarissa, Mater. Sci. Semicond. Process. 31, 124 (2015).

[19] N. Bouarissa, Physica B 406, 2583 (2011).
[20] P. Blaha, K. Schwarz, G.K.H. Madsen, D. Kvasnicka J. Luitz, "WIEN2k: An Augmented Plane Wave plus Local Orbitals Program for Calculating Crystal Properties", Tech. Universität, Wien 2001.

[21] P. Hohenberg, W. Kohn, Phys. Rev. 136, B864 (1964).

[22] Z. Wu, R.E. Cohen, Phys. Rev. B 73, 235116 (2006).

[23] H.J. Monkhorst, J.D. Pack, Phys. Rev. B13, 5188 (1976).

[24] S. Zerroug, F. Ali Sahraoui, N. Bouarissa, Eur. Phys. J. B 57, 9 (2007).

[25] S. Zerroug, F. Ali Sahraoui, N. Bouarissa, Appl. Phys. A 97, 345 (2009).

[26] R.W.G. Wyckoff, Crystal Structures, 2nd ed., Interscience, New York 1963, p. 17.

[27] H. Algarni, O.A. Al-Hagan, N. Bouarissa, M.A. Khan, T.F. Alhuwaymel, Spectrochim. Acta A 190, 215 (2018).

[28] Y. Harrache, N. Bouarissa, Solid State Commun. 295, 26 (2019). 